\title{
Las instituciones educativas y la comunidad frente al maltrato infantil: una experiencia de investigación acción participativa
}

\author{
María Dilia Mieles Barrera \\ María Victoria Gaitán Espitia \\ Universidad del Magdalena \\ Renán Cepeda Gaitán \\ Universidad Sergio Arboleda y Universidad Austral de Chile
}

\section{Resumen}

La investigación "Diagnóstico del Maltrato Infantil en la Comuna 8 del Distrito de Santa Marta”, financiada por el Fondo Patrimonial para el Desarrollo de la Investigación de la Universidad del Magdalena (Santa Marta, Colombia) y desarrollada por el Grupo de Investigación en Educación Infantil, caracteriza las formas de maltrato infantil (MI) más frecuentes en esta zona de la ciudad y describe el papel de las instituciones educativas y la comunidad frente a esta problemática. La metodología InvestigaciónAcción-Participativa (IAP) permitió movilizar la sociedad civil, instituciones educativas y organizaciones gubernamentales para trabajar en el diagnóstico y prevención. Se encontraron todas las formas de maltrato caracterizadas y los principales maltratadores se reconocieron como pertenecientes al grupo familiar. Se identificaron factores protectores en las familias, la comunidad y el Estado. La extrema pobreza y el bajo nivel educativo propician el maltrato y originan condiciones de vida injustas para la infancia, violación de sus derechos y grave daño en su desarrollo integral. Se recomienda: i) Desarrollar estrategias de formación y prevención con las familias y la comunidad para disminuir la violencia intrafamiliar y escolar; ii) que las instituciones educativas intervengan con una activa función preventiva; iii) organizar programas de apoyo social, económico y psicológico, que mejoren la calidad de vida de las familias y contribuyan a la protección de la infancia, con apoyo de universidades, organismos oficiales e institucionales, iv) difundir ampliamente los resultados visualizando la preocupante presencia del maltrato infantil y concientizando sobre su prevención.

\section{Palabras clave}

Correspondencia:

Maria Victoria Gaitán Espitia

mavigaes3@yahoo.es
Maltrato infantil [MI] - Prevención del maltrato - Promoción del buen trato. 


\title{
Educative institutions and the community faced with child maltreatment: an experience of participative action research
}

\author{
María Dilia Mieles Barrera \\ María Victoria Gaitán Espitia \\ University of Magdalena \\ Renán Cepeda Gaitán \\ Universidad Sergio Arboleda y Universidad Austral de Chile
}

\begin{abstract}
The study entitled "Diagnostic of Child Maltreatment in Commune 8 of the District of Santa Marta" (Diagnóstico del Maltrato Infantil en la Comuna 8 del Distrito de Santa Marta) sponsored by the Patrimonial Fund for the Development of Research of the University of Magdalena (Santa Marta, Colombia) and conducted by the Research Group in Child Education characterizes the most frequent forms of child maltreatment (CM) in this area of the town, and described the role of educative institutions and of the community in facing this problem. The methodology of Participative-ActionResearch (PAR) made it possible to mobilize the civil society, educative institutions, and government organizations to work in the diagnostic and prevention. All forms of maltreatment characterized were identified, and the main perpetrators were seen to belong to the family group. Protecting factors were identified in the families, in the community, and in the State. Extreme poverty and low levels of education are associated to maltreatment, and originate unfair living conditions for children, violations of their rights, and severe damages to their whole development. The study recommends: i) To develop strategies of formation and prevention with families and the community to decrease school and intra-family violence; ii) that educative institutions should intervene with an active preventive function; iii) to organize programs of social, economic, and psychological support that help to improve the quality of life of the families, and contribute to protect children, with the collaboration of universities, and official and institutional bodies; iv) to disseminate widely the results, exposing the worrying presence of child maltreatment, and helping to increase awareness of its prevention.
\end{abstract}

\section{Keywords}

Contact:

María Victoria Gaitán Espitia

mavigaes3@yahoo.es
Child maltreatment [CM] - Prevention of maltreatment - Promotion of child care. 
Para esta investigación se considera maltrato infantil [MI], lo definido en el Código de Infancia y Adolescencia (CN, 2007, Artículo 18):

Toda forma de perjuicio, castigo, humillación o abuso físico o psicológico, descuido, omisión o trato negligente, malos tratos o explotación sexual, incluidos los actos sexuales abusivos, la violación y en general toda forma de violencia o agresión sobre el niño, la niña o el adolescente por parte de sus padres, representantes legales o cualquier otra persona.

Es importante iniciar este artículo estableciendo la definición de maltrato válida para nuestro territorio, considerando que cualquier definición de MI se encuentra anclada en el sistema social y está relacionada con los acuerdos de una comunidad sobre las prácticas de crianza y educación aceptables y con las actitudes y valores que se promueven. Tal como plantea Garbarino (1986), el maltrato es intrínsecamente una etiqueta social, así que no basta con que una conducta sea nociva, sino que, además, debe violar alguna norma de aquello que se considera apropiado, de acuerdo con lo pactado por una sociedad. Es por ello necesario establecer una definición de MI que soporte la toma de decisiones relacionadas con la vida, la salud, el bienestar físico, psicológico y social de las personas, así como para el establecimiento de medidas preventivas y punitivas en un contexto sociocultural (BELSKY, 1980).

El MI afecta de manera devastadora la vida de niñas y niños que se convierten, por diversas circunstancias, en víctimas de padres, madres, adultos o pares, que los someten a todo tipo de vejámenes y abusos, dada su vulnerabilidad y dependencia. Aunque es un fenómeno de carácter global que ha estado presente en la vida infantil desde los albores de la civilización, hoy ha cobrado mayor visibilidad y se comprende de mejor manera su complejidad y carácter perturbador en lo personal, familiar y social. No obstante ser un fenómeno investigado sistemáticamente, aún continúa como una problemática enraizada en los procesos de crianza y en las tradiciones familiares.

Más allá del marco normativo y legal, cuando se hace referencia al MI se consideran una serie de conductas y acciones que son lesivas para los niños $\mathrm{y}$, por tanto se pueden establecer diferentes tipos de maltrato que tienen sus propias definiciones y características, lo que permite investigarlos ya sea de manera específica o de forma integrada, considerando que habitualmente no se ejerce un solo tipo, sino que el maltratador a la vez ocasiona diversos daños, que pueden ser físicos, emocionales, o de otra índole. Al hacer referencia a los tipos de MI es importante tener en cuenta que estas clasificaciones están enmarcadas en pronunciamientos de orden internacional y nacional: la Convención sobre los Derechos del Niño (ONU, 1989), el Código de Infancia y Adolescencia (CN, 2007) el Plan Nacional de Atención a favor de la Infancia, entre otros, en los cuales se concibe a niñas y niños como sujetos de derechos, reivindicando el papel activo de cada uno en su propio desarrollo, el de su familia y la comunidad.

En el MI se pueden diferenciar dos grandes modalidades: maltrato por acción y maltrato por omisión. En el primero, el maltratador provoca con su acción directa (no accidental) daños físicos (por ejemplo, golpes, quemaduras, sacudir o zarandear a un bebé, abuso sexual (MEJÍA, 1996; CASIÓN et al., 2001) o emocionales-psicológicos a través de la hostilidad verbal crónica en forma de insulto, desprecio, crítica o amenaza de abandono, y constante bloqueo de las iniciativas de interacción infantiles. Por otro lado, el maltrato por omisión se entiende como descuidar o dejar de atender las necesidades básicas (por ejemplo, higiene, salud, nutrición, educación, afecto) del niño o la niña (MARTÍNEZ; DE PAUL, 1993). Esto se reconoce como negligencia, la cual puede ser física, emocional y educativa (CASIÓN et al. 2001). 
A partir de los años 70, buena parte de la investigación ha estado dirigida a explicar la etiología del MI, lo que ha dado origen a varias perspectivas teóricas, que van desde la consideración de variables individuales denominadas modelos de factor único y clinico-psiquiátricos, hasta considerar los sistemas de interacciones que se producen entre la familia y los entornos en los que se encuentra inmersa (por ejemplo, modelos interaccionistas-ecosistémicos). De esta manera se ha avanzado desde una primera mirada intraindividual y unicausal basada en las características personales de los involucrados, identificando en los maltratadores características psicológicas y psicopatologías, vinculadas con desórdenes mentales y de personalidad (KEMPE; HELFER 1972; SPINETA; RIGLER 1972; MARTÍNEZSEGURA, 2003), a posiciones multicausales que tienen en cuenta aspectos económicos, sociales, culturales y ambientales presentes, en mayor o menor medida, en las situaciones de maltrato (KORBIN, 1980; MARTÍNEZ; ROIG, 1987; GARBARINO; KOSTELNY, 1992; ARRUABARRENA; DE PAUL, 1994; MARTÍNEZ-SEGURA, 2003; SORIANO, 2008).

En particular, las diversas líneas de investigación sobre MI en Latinoamérica, han sido orientadas hacia el entendimiento de factores socioeconómicos (ZUNZUNEGUI; MORALES; MARTÍNEZ, 1997), socioculturales e históricos (GRACIA et al., 1994; SANTANA; SÁNCHEZ; HERRERA, 1998; ARACENA et al., 2000; PARDO, 2003), sociopolíticos (MEN, 2007), conductuales (CABALLERO; LIÑÁN; MARTÍNEZ, 2001; GARCÍA, 2002) e incluso considerándolo como un problema de salud pública (VIZCARRA et al., 2001). Estos trabajos han permitido dilucidar patrones comunes entre diferentes países latinoamericanos, donde claramente el castigo físico es el comportamiento violento más común de $\mathrm{MI}$, el cual tiene profundas raíces socioculturales y está asociado principalmente con las comunidades más vulnerables, de poca escolaridad y de menor condición socioeconómica. Sin embargo, muy pocos trabajos académicos o gubernamentales han entregado pautas para la prevención del MI y la vinculación participativa de la comunidad más allá del contexto legal de cada país.

La presente investigación se basa en el aporte de Cicchetti y Rizley (1981), quienes consideran que para alcanzar una comprensión holística de las causas del MI, es necesario incluir tanto factores de riesgo como factores de compensación o protección, los cuales deben ser considerados simultáneamente, tanto en la familia como en la comunidad. Para tal fin se tuvo en cuenta la caracterización que se ha hecho de las modalidades del maltrato infantil en sus diversas manifestaciones, dado que para comprender este fenómeno y realizar intervenciones efectivas orientadas a su prevención, es necesario conocer los tipos de maltrato infantil y sus indicadores. En este sentido, el objetivo planteado fue identificar las formas y manifestaciones de maltrato que afectan la población infantil en la Comuna $8 \mathrm{del}$ Distrito de Santa Marta, a través del diagnóstico participativo, con organizaciones del Estado, instituciones educativas y la sociedad civil, para comprometer y consolidar el colectivo social e institucional, y desarrollar propuestas pedagógicas para la prevención del maltrato y promoción del buen trato a la infancia.

\section{Metodología}

La investigación es un estudio social de carácter cualitativo con un enfoque de Investigación Acción Participativa (IAP), que permite el abordaje del problema desde la comunidad y el planteamiento de propuestas de intervención generadas en la participación social, a partir de fuentes confiables y de los mismos actores e instituciones participantes. Los objetivos buscaron caracterizar las formas de maltrato infantil más frecuentes y generar acciones institucionales y comunitarias de prevención del MI y promoción del buen trato a los niños, involucrando a toda la comunidad. 


\section{Comunidad estudiada}

La investigación se realizó en la Comuna 8 de Santa Marta, Caribe Colombiano. Sus características socioeconómicas se enmarcan en extremos sociales de desigualdad, caracterizados por la presencia de un sector hotelero y residencial con alto nivel de vida y otro sector tugurial con extremos de pobreza (Alcaldía de Santa Marta, 1999). El estudio se centró en este último grupo, cuyas condiciones de desprotección generan un ambiente propicio para la intensificación del MI. Investigadas fuentes primarias y secundarias se identificó esta comuna como una de las que presentan mayor incidencia del MI (Casa de Justicia, 2008).

La población se integró con 116 niños y niñas pertenecientes a cinco instituciones educativas participantes (67 niños y 49 niñas) entre cinco y doce años, que los docentes identificaron con indicios de maltrato. Con ellos se organizaron grupos focales en cada escuela y se desarrollaron algunos talleres lúdicoreflexivos donde tuvieron la oportunidad de pensar sobre su vida, aprender a detectar y prevenir el maltrato y se explicaron pautas para mejorar las relaciones con su familia y en la escuela. Igualmente se aplicó un cuestionario para identificar las formas de maltrato más habituales. Con 23 (13 niñas y 10 niños), cuyos padres firmaron el consentimiento informado se hicieron entrevistas en profundidad, se les aplicó el test de familia y se brindó asesoría psicológica al núcleo familiar.

\section{Diagnóstico del MI}

Se realizó mediante una acción comunitaria con la participación de líderes cívicos, sociales y religiosos, las madres comunitarias, los docentes y directivos docentes, las familias y las organizaciones de salud. Se orientó a identificar las diferentes formas en que este problema se presenta en el sector, partiendo del principio que si bien, el fenómeno de violencia y MI tiene denominadores comunes, también es cierto que los factores asociados a la condición social y cultural definen características particulares que deben ser identificadas con el fin de desarrollar estrategias apropiadas para su prevención, que realmente sean aceptadas por la comunidad (SANTANA; SÁNCHEZ; HERRERA, 1998).

Para ampliar el diagnóstico se aplicó encuesta a una muestra intencionada de 450 habitantes de diversos sectores de la comuna. La encuesta se elaboró basada en la Guía para la detección del maltrato infantil (CFPE, 2002) incluyendo indicadores de maltrato para todas las formas conocidas. Permite caracterizar de manera general al encuestado, omitiendo su identidad. Las categorías utilizadas son: maltrato físico, maltrato emocional, negligencia, abuso sexual, síndrome de bebé zarandeado, y otras formas de violencia; las preguntas tienen opciones de respuesta cerrada y abierta. El instrumento fue sometido a una revisión de validez de constructo mediante la técnica de jueces expertos, prevía una prueba piloto con población de 50 personas, y el ajuste en la formulación de las preguntas.

Igualmente, se consultaron fuentes secundarias como Bienestar Familiar, Fiscalía, Defensoría del Pueblo, Comisaría de Familia, Casa de Justicia, iglesias, hogares comunitarios e instituciones educativas de preescolar y primaria de la Comuna.

\section{Indicadores de MI}

La caracterización del MI involucró la evaluación de indicadores claros que permitieran identificar las diversas formas de maltrato (por ejemplo, por acción, omisión, familiar y extrafamiliar). De esta manera los indicadores fueron definidos así:

1) Maltrato físico: Lesión física producida de manera no accidental que se manifiesta por moretones, enrojecimientos y/o fracturas resultantes de puños, patadas, mordiscos, pellizcos, quemaduras, palazos, corrientazos, inmersión en líquidos, presiones, entre otros.

2) Maltrato emocional: Dentro de esta modalidad de maltrato se encuentran numerosas conductas 
que padres, maestros, cuidadores o pares ejercen con los niños (por ejemplo, rechazo, crítica, constante burla de sus aptitudes o de sus errores y equivocaciones infantiles, ridiculización de sus acciones, amenaza de abandono o daño si no se cumplen las expectativas).

3) Abuso sexual: Cualquier clase de contacto sexual de un adolescente o adulto con un niño, donde el primero posee una posición de poder o autoridad sobre el niño, y cuyo fin es satisfacer las necesidades sexuales del abusador o de un tercero (MEJÍA, 1996; CASIÓN et al., 2001). El abuso sexual incluye una amplia gama de comportamientos y conductas, que determinan distintas formas: Contacto físico (por ejemplo, masturbación, tocamientos, besos, sexo oral, penetración vaginal o anal y otros), sin contacto físico (por ejemplo, hostigamiento o acoso sexual, exhibición de los genitales del adulto, compartir material pornográfico con el niño o utilizarlo para la elaboración de material pornográfico).

4) Síndrome del bebé zarandeado: Este síndrome se entiende como las lesiones cerebrales que se producen por sacudir o zarandear al niño menor de dos años. Las sacudidas severas, repetitivas, pueden producir hemorragias cerebrales afectándolo, sin que se evidencie lesión física externa; el niño puede llegar a convulsionar, presentar ceguera, alteraciones de la conciencia o inconsciencia. Su diagnóstico es difícil si no se hace oportunamente.

5) Negligencia: Privación de los elementos básicos necesarios para garantizar el desarrollo armónico e integral del niño: alimentación, salud, cuidado, afecto, entre otros. Puede ser temporal, asociada a un período o evento determinado como una enfermedad o permanente, como el abandono de un niño con discapacidad.

a) Negligencia física: Se presenta cuando el progenitor o adulto responsable deja de proveer al niño lo necesario para salvaguardar su integridad física, como la falta de cuidado por una lesión física, enfermedad o privación de atención médica adecuada y oportuna; falta de acompañamiento; dejarlos en manos de personas no calificadas para atender sus necesidades; inatención a situaciones de riesgo y peligros en casa; inadecuada nutrición, vestido e higiene y otras formas de descuidar su bienestar y seguridad.

b) Negligencia emocional: Se presenta cuando hay desatención de las necesidades afectivas y emocionales de los niños, negación del afecto, aislamiento del grupo familiar. La negligencia emocional se manifiesta cuando hay

falta persistente de respuesta a las señales, expresiones emocionales y conductas procuradoras de proximidad e interacción iniciadas por el niño, y falta de iniciativa de interacción y contacto, por parte de una figura adulta estable. (CASIÓN et al., 2001, p. 24)

En algunos casos se asimila al abandono emocional, cuando los padres conviven con los niños pero no están presentes para acompañar el desarrollo, consolar y permitir con su presencia y cercanía la construcción de una relación afectuosa, continua y permanente.

c) Negligencia Educativa: Se refiere a favorecer o propiciar la deserción escolar, no matricular al niño en la escuela; oponerse para que obtenga servicios educativos alternos en casos de necesidades educativas especiales.

\section{Factores de riesgo}

Se considera que existe un elevado número de situaciones que predisponen al maltrato, que se clasifican en tres grandes grupos (SORIANO, 2005):

a) Factores individuales o personales: tanto de los padres como de los niños, que pueden ir desde las características físicas, la personalidad, las experiencias anteriores de crianza vividas por los padres, hasta la inexperiencia de éstos. 
b) Factores familiares: Referidos a la estructura de la familia, al funcionamiento y la dinámica de ésta. Algunos son: la existencia de padres adolescentes, hijos no deseados, familias monoparentales, violencia familiar, malas relaciones y comunicación, carencia de vínculos afectivos e inexistencia de límites o reglas familiares (GARCÍA, 2002). c) Factores socioculturales y ambientales, se distinguen: la situación laboral (por ejemplo, desempleo, pobreza, inestabilidad, economía sumergida, prácticas marginales) Vivienda (por ejemplo, hacinamiento, malas condiciones habitacionales). Las relaciones sociales (por ejemplo, aislamiento, ausencia de participación comunitaria), cultura (por ejemplo, rechazo $\mathrm{y}$ actitudes arraigadas de violencia hacia la infancia, la mujer y la paternidad).

Cuando se producen situaciones de maltrato, concurren más de uno de estos factores, por lo cual se considera una problemática multicausal (SORIANO, 2008).

\section{Análisis y tratamiento de la información}

Identificados los tipos de maltrato más comunes se formularon, de manera colectiva, propuestas pedagógicas de promoción del afecto y el buen trato para los niños, respaldadas por las instituciones educativas, gubernamentales y la comunidad participante. Se buscó generar soluciones basadas en información concreta, permitiendo iniciar procesos para la prevención del problema y sus manifestaciones, concientización y consolidación de la comunidad y las instituciones en la búsqueda de estrategias favorables al aprendizaje de formas de relación basadas en el afecto y el buen trato y la solución activa pero pacífica de los conflictos, en un contexto de permanente apoyo, asesoría y acompañamiento del proceso de diagnóstico y la formulación de las propuestas de intervención en las que se comprometieron todos los actores sociales convocados y en particular, las instituciones educativas.
La metodología incluyó socialización de la investigación y los hallazgos emergentes con todas las personas comprometidas en ella; 1.211 personas participaron con el fin de avanzar en la concientización sobre el problema y mejorar los procesos de crianza a través de encuentros comunitarios y talleres de formación. Adicionalmente, buscando integrar más a la comunidad y sensibilizarla, se realizaron actividades lúdicas de integración y socialización familiar y comunitaria como festivales, vacunación contra el maltrato, celebración del día del niño, salida a la playa. En estos espacios se identificaron y fortalecieron los factores protectores presentes en la comunidad.

Las instituciones educativas fueron consideradas en la presente investigación como epicentros del trabajo, debido al potencial que tienen como espacio aglutinador de actores sociales de las comunidades, aprovechando las nuevas responsabilidades establecidas para éstas en el Código de Infancia y Adolescencia, referidas a funciones de prevención, protección y denuncia frente al maltrato infantil.

La información se procesó con los programas estadísticos, Statistic y PAST.

\section{Resultados}

$\mathrm{Al}$ iniciar la recolección de información y detectar resistencia en la comunidad para identificar casos de MI se hicieron cambios en la secuencia metodológica y se motivó el desarrollo de proyectos para prevención del maltrato y promoción del buen trato en las instituciones educativas y en los hogares comunitarios. El mayor conocimiento del tema, aceptarlo como un problema arraigado en la comunidad y la generación de un clima de confianza, favoreció que espontáneamente fueran surgiendo historias de niños y niñas maltratados, algunas de las cuales fueron denunciadas por habitantes de la comuna.

\section{Caracterización del MI}

Los resultados permiten hacer una descripción de las formas de MI comunes en esta 
población, diagnosticar la gravedad del problema, planear y realizar proyectos de prevención del maltrato y promoción del buen trato a la infancia. Dentro de las formas frecuentes de MI identificadas, se destacan la violencia intrafamiliar (Figura 1), maltrato físico con diversas modalidades (Figura 2), y el maltrato emocional (Figura 3). Igualmente se logró obtener una imagen de la percepción sobre otras formas de MI presentes en esta comunidad (Figura 4).

Figura 1: Maltrato físico. Violencia física familiar con los niños y niñas.

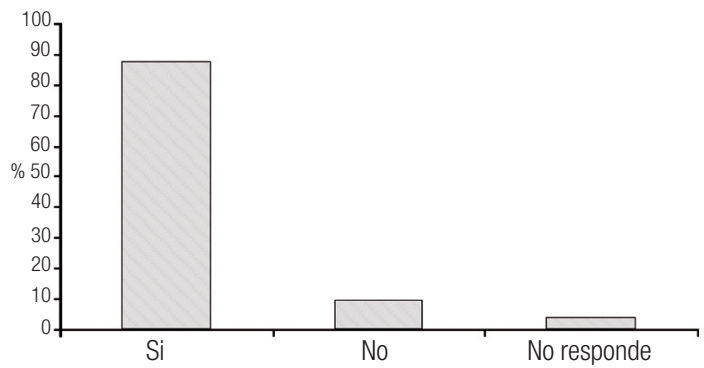

Ante la pregunta "¿En tu casa te pegan?" $87 \%$ de los niños entrevistados respondieron afirmativamente, evidenciando en alto grado la presencia del maltrato físico en los hogares.

Resultados con niños: La información hizo posible caracterizar el maltrato, desde la percepción y vivencia infantil. En un taller en que se indagó cómo es un día habitual en la familia, pidiéndoles, según la edad, que lo hicieran con relatos o dibujos, se encontraron diversas situaciones siendo las más comunes los gritos y peleas desde la mañana hasta la noche, la mamá hace los oficios de la casa, y el papá a veces trabajando y también jugando dominó o mirando tv, los hermanos peleando, jugando en la calle o haciendo mandados.

La presencia de peleas en el hogar es significativa (87\%). Expresiones como ésta: "imis papás no pelean, se quieren matar, agarran hasta cuchillo!”, se escuchan con frecuencia. El porcentaje que se presenta en la categoría de maltrato físico (Figura 1), indica que en los hogares se viven y resuelven conflictos expresados en peleas violentas, lo que es imitado por los niños en la vida diaria. Se utiliza como estrategia de corrección el golpearlos con objetos característicos como correas, varas, zapatos, la mano, entre otros, por motivos como no obedecer, agredirse entre hermanos, salir a la calle u otras circunstancias ajenas a ellos como consumo de alcohol por parte de familiares y frustraciones personales entre otras.

Figura 2: Modalidades del maltrato físico:

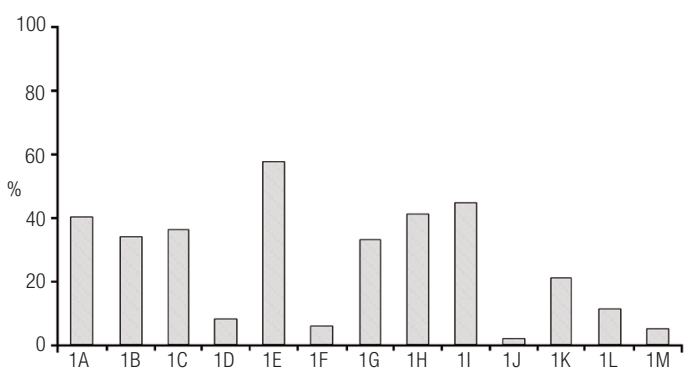

1 A: golpes con la mano, el pie en la cara. 1 B: Golpes con la mano, el pie en el cuerpo. 1 C: Lanzarle objetos. 1 D: Quemaduras. 1 E: Latigazos, rejazos, correazos, chancletazos. 1F: Chapuzón en líquidos o lanzamiento de estos. 1 G: Pellizcos. 1 H: Empujones. 1 l: Halones de oreja, cabello, brazos. $1 \mathrm{~J}$ : Corrientazos. $1 \mathrm{~K}$ : Presiones fuertes. $1 \mathrm{~L}$ : Dejarlo sin comer. $1 \mathrm{M}$ : Otras modalidades no incluidas. (El número 1 corresponde a la posición de la pregunta en la encuesta).

Al aplicar el test de familia, con un niño de seis años y preguntarle ¿quiénes están en el dibujo? ¿Quiénes son los buenos y los menos buenos? Hablaba así:

el papá viene a coger a la mamá para pegarle y la mamá le quiere pegar al hijo... el más bueno es el hijo, el menos bueno el papá....es malo...pega con correa en la pierna... (hizo el gesto como le pegaba).

Durante una asesoría psicológica a un niño de seis años, que frecuentemente era castigado por la maestra, por agresiones recurrentes a sus compañeros, relató lo siguiente: 
papá se fue allá abajo (tiene una novia), bebe ron, pelea, juega billar...busca pescado... yo peleo con los niños, mi mamá no me da plata para comprar... peleo en el salón...mi mamá me pega con la correa...me pega en la cara con la mano...me tiró al piso...todos los días me pega porque peleo. Me gusta jugar, portarme mal en el colegio y bien en la casa... ir a la playa...agarrar pescaitos, ver televisión. Mi papá le pega a mi mamá con un palo en la espalda...mi mamá agarra también un palo y le lanza una piedra...yo le pego con piedra también a él...

Figura 3: Modalidades de maltrato emocional infantil.

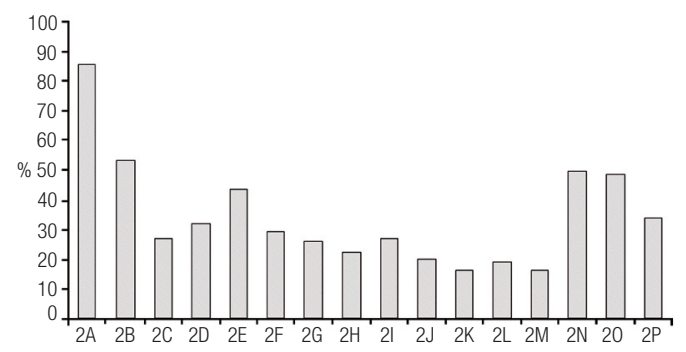

2 A: Gritos. 2 B: Insultos. 2 C: Rechazo. 2 D: Crítica constante. 2 E: Palabras ultrajantes y que disminuyen su autoestima. $2 \mathrm{~F}$ : Burla de sus características físicas. $2 \mathrm{G}$ : Burla de sus comportamientos y errores. $2 \mathrm{H}$ : Amenaza de abandono o daño. 2l: Oficios, tareas y exigencias inapropiadas para la edad. $2 \mathrm{~J}$ : Amenazas constantes de castigos severos (encerrarlos, regalarlos, abandonarlos). $2 \mathrm{~K}$ : Hacerlos sentir culpables de los problemas familiares. 2 L: Dejarlos encerrados. $2 \mathrm{M}$ : Echarlos a la calle. $2 \mathrm{~N}$ : Prohibirles jugar o hacer cosas que les gusten. 2 0: Protegerlo demasiado. $2 \mathrm{P}$ : Dejarlo hacer lo que quiere. (El número 2 corresponde a la posición de la pregunta en la encuesta).

El maltrato emocional se evidencia porque los niños son agredidos verbalmente por parientes con palabras ultrajantes, maltratándolos emocionalmente en un 78\%. Gritos e insultos que deterioran su autoestima y personalidad los acompañan en su vida familiar y aún en las instituciones educativas, como un medio para establecer disciplina y obediencia. Dos hermanitas de 8 y 10 años viven con su padre porque su madre las abandonó, comentan que

su padre las golpea... a veces les muerde las orejas cuando salen a la calle a jugar... les dice cosas horribles... que se vayan de la casa, que busquen marido... que está muy aburrido con ellas... que un día de estos se va a ir y no vuelve más... etc.

Las niñas y niños están expuestos a negligencia o abandono por parte de los adultos encargados de su crianza. El 60\% permanecen solos debido al trabajo y/o a la búsqueda de recursos para el sostenimiento diario, o por otras razones. Se evidencia en el aspecto físico y presentación personal muy descuidada en la que es común encontrar piojos, caries, problemas de salud, circunstancia que se da por negligencia de los padres o porque carecen de registro civil, requisito para acceder a los servicios de salud. Se trabajó con una niña de siete años, que se queda sola en casa, porque la mamá sale todos los días, (dice que a jugar dominó), ella tiene que preparar la comida de las dos y hacer todos los oficios de la casa, también cuidar a un hermanito que tiene dos años, a la tarde va al colegio y hace sus tareas sola.

Se identificó maltrato por negligencia educativa, emocional y en salud. El 51\% no tienen acompañamiento para la realización de actividades diarias como aseo, alimentación, trabajos escolares, apoyo en el proceso educativo, siendo reemplazados los padres, en algunos casos, por otros familiares, vecinos y amigos.

$\mathrm{Al}$ indagar acerca del abuso sexual, en la aplicación de un taller denominado "caricias buenas y caricias malas (por ejemplo, partes íntimas)", se encontró que las niñas y niños ven este tema como tabú, mostrándose reacios a exponer qué entienden por abuso sexual, muchos manifestaron: “me da pena decir...". La mitad de la población identifica algunas formas de abuso sexual. Sin embargo, existe un alto porcentaje de desconocimiento o concepto distorsionado sobre el mismo, lo cual hace imperativo trabajar este aspecto, porque muchos comportamientos abusivos son vividos como normales.

Además del maltrato, también experimentan carencias en aspectos vitales como alimentación, vestido, recreación y útiles escolares. 
Resultados con adultos: Coincidiendo con los niños, los adultos identificaron el maltrato físico en todas sus formas como la modalidad más frecuente de MI, utilizado por el grupo familiar (Figura 2). Le sigue el maltrato emocional con gritos e insultos, burlas, amenazas constantes de castigos y otras modalidades (Figura 3). El sindrome del niño zarandeado es desconocido como una forma de maltrato. Comprender su gravedad generó reflexión en los adultos, quienes manifestaron que acostumbran lanzar el niño al aire en forma de juego o tomarlo bruscamente, ignorando el daño cerebral que esto puede causar.

Figura: 4 Percepción sobre otras formas de maltrato:

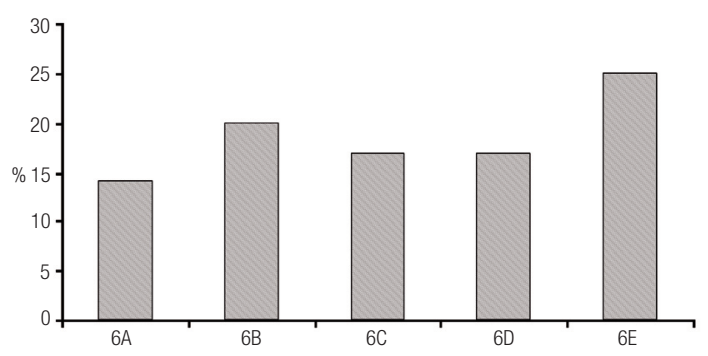

6 A: Prostitución. 6 B: Alcoholismo. 6 C: Drogadicción. 6 D: Mendicidad. 6 E: Trabajos inapropiados. (El número 6 corresponde a la posición de la pregunta en la encuesta).

En la percepción sobre otras formas de maltrato (Figura. 4), manifestaron conocer niños que realizan trabajos inapropiados para la edad, casos de alcoholismo infantil, drogadicción y mendicidad. El alcoholismo presente desde edades tempranas agrava la problemática infantil y se relacionaron algunos casos de prostitución, difíciles de identificar por múltiples temores de la comunidad.

\section{Participación activa de la comunidad y las instituciones}

La investigación permitió obtener información veraz sobre la situación en los diversos estamentos que participaron, generar reflexión y acción colectiva en torno al proble- ma, vislumbrar y definir alternativas de solución y prevención que permitan la protección de la infancia. Un total de cinco instituciones educativas de la comunidad participaron en la construcción y desarrollo de proyectos institucionales para la prevención del maltrato y promoción del buen trato a los niños. Cada institución trabajó en torno a la consigna que identificó su proyecto con un nombre, que a la vez fue el objetivo del mismo: CED (Centro Educativo Distrital) Aeromar: "Un encuentro con La Ternura"

CED Don Jaca: "Sembremos Lazos de Amor"

IED (Institución Educativa Distrital) Jesús Espeleta Fajardo: "Con amor y ternura corazoncitos alegres" IED Cristo Rey: "Unidos por el buen trato"

CED Bellavista: "Creciendo juntos con respeto y amor"

Líderes y madres comunitarias: "Unidos por el buen trato".

En estos proyectos trabajados con el método de talleres de formación en valores, desarrollo personal, relaciones familiares; derechos, legislación y protección a la infancia, surgió la motivación para incluirlos en el Proyecto Educativo Institucional (PEI) de cada centro educativo, para su desarrollo anual.

Estos proyectos, que se espera sean institucionalizados de forma permanente, tienen características específicas: son construidos integral y participativamente en las instituciones educativas; previamente, se realiza un diagnóstico institucional y acuerdos sobre necesidades particulares de cada comunidad educativa; los proyectos se promocionan y desarrollan simultáneamente a través de talleres con los niños, padres, madres y cuidadores, con la asesoría de docentes y personas especializadas; se planean a largo plazo con evaluaciones periódicas y gestión de recursos humanos, financieros y logísticos para su desarrollo.

No obstante, y a pesar de la buena disposición encontrada para la participación institucional, el trabajo de campo demostró que en algunos casos, se desconoce por completo el tema del MI, se tiene poco interés en abordarlo y no se está cumpliendo el mandato legal. Frente a 
la amplia invitación que se hizo a los docentes para participar activamente en el proyecto, muchos desistieron aduciendo "excesivo trabajo y no querer buscarse problemas adicionales con padres que son bastante violentos". Estos temores que son reales y que inciden de manera negativa en la prevención del maltrato, fueron tenidos en cuenta para lo cual se hizo invitación a representantes de las instituciones oficiales creadas a partir de la Ley de Infancia y Adolescencia (CAVIF: Centro de atención integral a víctimas de violencia intrafamiliar; CAIVAS: Centro de atención integral a víctimas de abuso sexual), las cuales enviaron delegados especializados quienes explicaron en talleres y seminarios a los maestros y padres, las formas seguras de denunciar y actuar frente al MI y las oficinas, donde se atienden estos problemas preservando el anonimato.

\section{Discusión}

La identificación de características psicológicas, vinculadas con desórdenes mentales y de personalidad de los maltratadores (por ejemplo, modelos de factor único) al igual que la consideración de aspectos económicos, sociales, culturales y ambientales presentes, en mayor o menor medida, en las situaciones de maltrato (por ejemplo, modelos multicausales), permiten avanzar y entender la complejidad del MI en todas sus dimensiones. En este sentido, el presente trabajo no solo caracterizó la problemática presente en la comunidad bajo estudio utilizando un enfoque multicausal, sino que también buscó integrar a la comunidad e instituciones estatales y educativas, mediante la participación activa en la caracterización, el entendimiento y la búsqueda de mecanismos de prevención del MI.

Desde esta investigación se considera que padres y madres de familia, líderes comunitarios, sociales y politicos, maestros y directivos docentes, así como los niños y niñas, cumplen un papel trascendental en los procesos de prevención y detección del problema, debido al conocimiento cercano de este tipo de situaciones presentes en la comunidad. Sin embargo, durante el desarrollo de la investigación se pudo evidenciar que para muchos el MI no constituye un problema, pues lo consideran una forma habitual de relación con los pequeños, y otros a pesar de que reconocen la magnitud del fenómeno y la importancia de erradicar estas prácticas, en su labor cotidiana, tienen escasa incidencia en aspectos cruciales como la prevención, detección y denuncia de casos de maltrato, aun habiendo sido informados por los propios niños de situaciones críticas que afectan su vida. Igualmente, en el desarrollo de la investigación, las principales dificultades en la identificación y caracterización del MI se derivaron de la desconfianza de los adultos, aún de docentes, para evidenciar el problema y vincularse al proceso, en parte por la escasa credibilidad frente al sistema de justicia del Estado y por temor a represalias de los actores involucrados.

Infortunadamente, en Colombia, a diario se tiene referencia de alguna situación violenta que involucra a los niños como víctimas de MI, aún desde antes de nacer y aunque se han realizado grandes y continuos esfuerzos para prevenir y disminuir esta grave problemática, el creciente número de denuncias pone en evidencia que no son suficientes los esfuerzos que se hacen para su prevención y erradicación, misión en la que debe estar comprometida toda la sociedad (MEN, 2007). Esta situación ha alcanzado límites alarmantes por la violencia social, los desplazamientos, las condiciones de pobreza y miseria que propician el hacinamiento, el poco acceso a la educación, el desempleo, el consumo de sustancias psicoactivas y problemas asociados, que se convierten en factores de riesgo que predisponen al maltrato infantil (DANE, 2005). El Caribe Colombiano, y en particular Santa Marta, no son ajenos a lo que ocurre en el país y en Latinoamérica.

\section{Diagnóstico y caracterización del MI}

El hecho que todas las formas de MI tipificadas sean habituales en la comuna evidencia el impactante grado de violencia que 
caracteriza las relaciones intrafamiliares en esta comunidad y por ende los nefastos efectos sobre el desarrollo de la personalidad y la capacidad para establecer relaciones armónicas y enriquecedoras con las demás personas, pudiéndose considerar y manejar el MI como un problema de salud pública (VIZCARRA et al., 2001). Se identificó la agresión como un medio utilizado para solucionar problemáticas presentes en la familia y el establecimiento de roles de autoridad. Es significativo que estas formas de castigo se dan como un estilo natural de imponer autoridad por parte de los adultos responsables del cuidado de los niños. Se evidencia un grave problema relacional en el seno familiar y en un contexto sociocultural específico que coincide con los hallazgos de las investigaciones desarrolladas por SantanaTavira, Sánchez-Ahedo y Herrera-Bastos (1998) y Ochoa (2004), quienes consideran que gran parte de los problemas del niño, se ven reflejados en la vida adulta, debido a que durante el desarrollo ontogénico, la herencia que los padres traen consigo, ha sido influenciada por aspectos relacionados con su propia experiencia de crianza, configuración de la personalidad, carencias afectivas, frustraciones y otras particularidades de su historia personal que condicionan en buena medida el desempeño parental hacia los hijos (VIZCARRA , 2001; GARCÍA, 2002; MARTÍNEZ-SEGURA, 2003).

El abandono o negligencia por parte de los adultos encargados de la crianza se debe a la extrema pobreza que origina condiciones de vida injustas para la infancia por la desigualdad, la falta de equidad, justicia social, generando un ambiente propicio para la violación de todos sus derechos. Aunque existe legislación pertinente por parte del Estado, las condiciones reales del entorno familiar propician todas las formas de maltrato, lo que nos lleva a reflexionar que sólo una política social equitativa e incluyente, aunada con una legislación pertinente, pueden propiciar ambientes de paz, convivencia y buen trato a la infancia.
Los niños y niñas de esta comunidad, sin protectores adultos en el seno familiar y muchas veces tampoco en las instituciones educativas, crecen indefensos en un mundo terriblemente violento ante su fragilidad y desprotección con las gravísimas consecuencias ya conocidas en los procesos de identidad, desarrollo de la personalidad, cognición, integración social y capacidad para construir la vida plena y feliz a la que tienen derecho (OGBURN; NIMKOFF, 1980). El trabajo cercano con los niños y niñas evidenció que dolorosamente su vida está atravesada por problemáticas que ponen en alto riesgo su integridad física, psicológica y social. Los maltratadores son personas cercanas y no cuentan con redes de apoyo que les permitan encontrar alguna salida a la situación presente.

\section{Consideraciones y problemáticas del MI}

Es importante considerar de acuerdo con Cuadros y Ordoñez, (2006), que las consecuencias negativas del MI y sus manifestaciones son heterogéneas, así un significativo número de víctimas de maltrato manifiestan problemas y otras logran superar esas experiencias adversas; algunas consecuencias se pueden presentar de manera inmediata y otras con el transcurso del tiempo afectando la capacidad para tener una vida plena. Esta situación es altamente preocupante si tenemos en cuenta que diversas investigaciones sobre maltrato en edad escolar han demostrado, reiteradamente como los niños que padecen maltrato manifiestan un funcionamiento comportamental problemático (ONU, 2006) o conductas de agresividad verbal y física, hostilidad, robos, mentiras, ausentismo escolar, que se integran en la categoría problemas de conducta o externalizantes (MEJÍA, 1996). Las manifestaciones de dichas conductas por parte de niñas y niños llevan a intensificar el maltrato en el seno del hogar, en la escuela y en la comunidad, lo cual convierte el problema en un círculo vicioso que pone en peligro la integridad física, emocional, 
psicológica y cognitiva de las víctimas $\mathrm{y}$ perpetúa la trasmisión intergeneracional del maltrato (CEREZO, 1995; SANTANA-TAVIRA; SÁNCHEZ-AHEDO; HERRERA-BASTOS, 1998).

Como en otras investigaciones (CUADROS; ORDÓÑEZ, 2006; ONU, 2006), se identifica a los padres, parientes, cuidadores adultos y hermanos como los principales maltratadores. Los abusadores sexuales también se ubican en el grupo familiar, convirtiendo así la familia, el primer lugar de protección para niños y niñas, en su mayor amenaza, con todo lo que implica para el aprendizaje de roles y desarrollo de la personalidad, resultados similares a los hallados por la Consulta Latinoamericana sobre la Violencia contra los Niños ONU, FNUI (2006). Es un patrón recurrente no solo en Colombia, sino también en países como España (ZUNZUNEGUI; MORALES; MARTÍNEZ, 1997), México (SANTANA-TAVIRA; SÁNCHEZAHED0; HERRERA-BASTOS, 1998), entre otros (ONU, 2006). Las formas de maltrato físico y emocional se presentan como fuertes características en los estilos de crianza de las familias y, en algunos casos, en la formación brindada en las instituciones educativas, lo cual no difiere significativamente de otros estudios (MEJÍA, 1996). Es muy grave la generalización de estas costumbres y su enraizamiento en las relaciones intrafamiliares y escolares, que llegan a convertirlas en la forma habitual de intercambio entre adultos y niños (MIELES; HENRÍQUEZ; SÁNCHEZ, 2009).

Es común que en situaciones de maltrato los niños no sean capaces de hablar del tema. Se sienten indefensos, desprotegidos y temerosos ante la amenaza que para ellos suponen los adultos e incluso en algunos casos llegan a creer que son los causantes de esta situación y por tanto la merecen (SORIANO, 2008). Por otra parte, sienten que en caso de denunciar los hechos, se dará mayor credibilidad a un adulto frente a su propio relato. No obstante, como fue evidente durante la investigación, los niños pueden avisar que algo va mal en su vida a través de un lenguaje no verbal, que se manifiesta mediante conductas tales como irritabilidad, mutismo, agresividad, pequeños hurtos, bajo rendimiento académico; además pueden manifestarlo a través de los juegos y dibujos, y en general todas aquellas conductas que signifiquen una llamada de atención o una reproducción de modelos paternos.

\section{Análisis de factores de riesgo en el MI}

Los principales factores de riesgo asociados al MI son la extrema pobreza, la violencia y desintegración familiar, la apatía, temor y desconfianza del adulto en las instituciones del estado para ejercer justicia en estos casos, que sumergen a la infancia de esta comunidad en un mar de sinsabores y agresiones, con traumáticos efectos en su vida presente y futura, situación agravada aún más por la violencia del desplazamiento forzado y vivencias de la guerra que muchos de ellos han experimentado. Adicionalmente, la falta de capacitación de las comunidades en el reconocimiento y respeto del otro, el aprecio por la vida, la participación y la organización como formas de superar sus actuales condiciones, la ausencia de referentes sociales y proyectos de vida, desconocimiento de los recursos jurídicos de protección a la infancia y la familia, se convierten en circunstancias que generan un ambiente propicio para la intensificación del MI y la violación de los derechos de los niños.

La existencia de condiciones de pobreza, desigualdad e injusticia, asociadas al MI, requieren de manera urgente la organización de programas de apoyo social, económico y psicológico, que mejoren la calidad de vida de estas familias y contribuyan a la protección de la infancia. Se coincide así con los resultados de la investigación de Zunzunegui, Morales y Martínez (1997) sobre estos factores de riesgo sociales y económicos en relación al MI. En esta perspectiva, teniendo en cuenta los factores 
de riesgo y las diferentes manifestaciones de MI, se considera entonces la prevención como una actividad prioritaria que debe responder a la complejidad del fenómeno, requiriendo un abordaje interdisciplinario, desde la participación de profesionales de distintas áreas, hasta el compromiso de agentes significativos de la comunidad.

\section{El rol de las instituciones educativas en la prevención del MI}

Sobre las instituciones educativas recaen múltiples responsabilidades frente a diversos problemas sociales que desbordan su capacidad. A pesar de las dificultades, la escuela es considerada un lugar propicio para abordar el problema del MI, detectarlo y prevenirlo por la cercanía que se vive entre docentes y estudiantes, por el tiempo que los pequeños pasan allí; porque a través de diversas actividades como las clases, el juego, las relaciones entre pares, se pueden evidenciar miedos, temores, comportamientos que no son habituales, alteraciones en la apariencia física, actitudes de hostilidad, poca capacidad para establecer vínculos afectivos. En este sentido, a la escuela se le reconoce, su poder de convocatoria frente a los padres y madres de familia o cuidadores, la credibilidad en el trabajo que desarrollan muchos maestros, que son admirados y respetados por la comunidad educativa, la posibilidad de impulsar procesos de aprendizaje en períodos relativamente largos e incorporarlos de manera sistemática a los proyectos educativos institucionales para hacerlos parte de la cultura escolar. Por consiguiente, es necesario considerar que para desarrollar exitosamente su función preventiva, la escuela como institución debe ser capaz de revisar sus propias actitudes y responsabilidades hacia el control de las conductas del MI (CN, 2007) y reconocer que en casos de maltrato se requiere un tratamiento integral no sólo de la víctima sino también de su agresor y de la familia, para lo cual se hace necesario gestionar ante las dependencias gubernamentales pertinentes el nombramiento o asignación de personal especializado para atender los casos que se presenten en la institución educativa o en la comunidad.

Por las razones expuestas, se recomienda desarrollar estrategias de formación y prevención con las familias y la comunidad para disminuir la violencia en el seno del hogar, en la escuela y los espacios comunitarios. Que las instituciones educativas universitarias intervengan con una función preventiva frente a esta problemática, estableciendo centros pilotos para prácticas de los programas de docencia, psicología, derecho y salud, cuyo trabajo se desarrolle alrededor de los temas y problemas de la infancia y la familia. Una estrategia de prevención necesaria, y pertinente en concordancia con los resultados de la presente investigación y los hallados por Gracia, Musitu, García y Arango (1994), es la de organizar proyectos institucionales que generen apoyo psicológico y social a las familias para prevenir el MI y capacitarlas para actuar participativamente en el análisis y búsqueda de soluciones a los problemas que forman parte de su vida cotidiana y en particular de las relaciones entre padres e hijos.

\section{Conclusiones}

Es absolutamente preocupante para la sociedad el incremento de todas las formas y manifestaciones del MI presentes en la Comuna 8 por las graves consecuencias que ello tiene en la construcción del tejido social y en el establecimiento de una cultura de violencia, con devastadores efectos en el desarrollo humano. Niños y adultos reconocen por igual la presencia de esta problemática en la comunidad, confirmándose así lo expuesto por Barudy (1998) sobre los graves efectos del MI en el desarrollo físico, psicológico, emocional y social de los niños afectados. Si bien es cierto que se logró sensibilizar a un significativo 
número de habitantes de la comuna sobre la gravedad del problema, es una comunidad que necesita constante apoyo para lograr un cambio real hacia el buen trato a los niños y la defensa de sus derechos. Aunque existen factores protectores en la comunidad, deben ser fortalecidos o desarrollados vinculando activamente a todas las familias.

Finalmente, es necesario destacar que los factores protectores para la promoción del buen trato y prevención del MI en la Comuna 8 de Santa Marta son: i) el incremento del compromiso de los padres de familia con la protección de los niños y la defensa de sus derechos; ii) la adquisición de mayor conciencia e integración de las instituciones educativas frente a las familias y el compromiso con la solución del problema; iii) el sentimiento creciente de comunidad y necesidad de asociación vivido y expresado por la población, sumado a la concientización sobre el problema y conocimiento de los procedimientos legales e instituciones para su atención; iv) un marco legal nacional fuerte y bien estructurado en defensa y promoción de la infancia, como también la organización y funcionamiento de instituciones gubernamentales responsables de su cuidado y protección.

Se espera que en un corto plazo, a partir de la movilización social generada desde la investigación y con la continuidad de los proyectos de intervención educativa, se evidencie una mayor sensibilidad y concientización sobre el tema que se traduzca en una reducción del MI en este sector de la ciudad. 


\section{Referencias}

ALCALDÍA DISTRITAL DE SANTA MARTA. Plan de Ordenamiento Territorial 2000-2009. Documento en línea. Disponible en: <http://www.santamarta.gov.co/index.asp?id_seccion=175\&id=1\&tisec=P.0.T>. Consulta: 15/03/2008.

ARACENA, Marcela et al. Resiliencia al maltrato físico infantil: variables que diferencian a los sujetos que maltratan y no maltratan físicamente a sus hijos en el presente y que tienen una historia de maltrato físico en la infancia. Revista de Psicología, Santiago, Chile, v. IX. ISSN (VI): 0716-8039, p. 3-8; 16-18, 2000.

ARRUABARRENA, María Ignacia; DE PAUL, Joaquín. Maltrato a los niños en la familia: Evaluación y tratamiento. Madrid: Pirámide, 1994.

BARUDY, Jorge. El dolor invisible de la infancia: una lectura ecosistémica del maltrato infantil. Barcelona: Paidos Ibérica, 1998.

BELSKY, Jay. Child maltreatment: an ecological integration. American Psychologist, Washington, v. 35, p. 320-335, 1980.

CABAlLERO, Elvia Rosa.; LIÑÁN, Carmen.; MARTínEZ, Nancy. Características Psicosociales de Familias de Menores Maltratados en el Municipio de Plato, Magdalena. Bogotá: UNICEF, 2001.

CASA DE JUSTICIA. Estadística denuncias de maltrato infantil. Documentos oficiales. Santa Marta, 2008.

CASIÓN, Manuel José et al., Guía para Detectar, Notificar y Derivar situaciones de Maltrato Infantil en Aragón. Zaragoza: IASS, 2001. Disponible en: <http://www.unav.es/icf/main/Planfamilia/aragon/Aragon_Guia_maltratoinfantil. pdf>. Consulta: 25/06/2010.

CEREZO, María Ángeles. El impacto psicológico del maltrato: Primera infancia y edad escolar. Revista Infancia y Aprendizaje, Valencia, ISSN: 0210-3702. v. 71, p. 135-157, 1995.

CICCHETTI, Dante.; RIZLEY, Ross. Development perspectives on the etiology, intergenerational transmission and sequela of child maltreatment. New Directions for Child Development, San Francisco, California, v. 11, p.11, 31-35, 1981.

CN. CONGRESO NACIONAL de Colombia. Ley 1098 de 2006. Código de la Infancia y Adolescencia. Bogotá: Imprenta Nacional, 2007

CFPE-Consellería de familia e promoción do emprego, muller e Xuventude, Xunta de Galicia. Guía para a detección do maltrato infantil. España, 2002.

CUADROS, Isabel; ORDÓÑEZ, Martha. La infancia rota. Bogotá: Norma, 2006.

DANE-Departamento Administrativo Nacional de Estadística. Censo nacional de población 2005. Bogotá, 2005. Documento en línea. Disponible en: <http:// www.dane.gov.co/censo/>. Consulta: 15/12/2008.

GARBARINO, James. Can we measure success in preventing child abuse? Issues in policy, programming and research. Child Abuse and Neglect journal, Chicago, v. 10, p. 143-156, 1986.

GARBARINO, James; KOSTELNY, Kathleen. Child maltreatment as a comunity problem. Child Abuse and Neglect journal, Chicago, v. 16. p. 465-464, 1992.

GARCÍA Enrique. Maltrato infantil en el contexto de la conducta parental: Percepciones de padres e hijos. Psicothema, Valencia, v. 14, n. 2, p. 274-279, 2002.

GARCIA, Enrique et al., Apoyo social y maltrato infantil: Un estudio en España y Colombia. Revista Interamericana de Psicología, Madrid, v. 28, n. I, p. 13-24, 1994. 
KEMPE, Henry.; HELFER, Ray. Helping the battered child and his family. Philadelphia: J. B. Pippincott, 1972.

KORBIN, Jill E. The cultural context of child abuse and neglect. Child Abuse and Neglect journal, Chicago, v. 4, p. 3-13, 1980.

MARTÍNEZ-ROIG, Alex. Paper de l'hospital en el diagnòstic i prevenció dels maltractaments. Fer Ciutat, Barcelona, v. 16, p. 23-27, 1987.

MARTínEZ-Roig, Alex.; DE PAUL, Joaquín. Maltrato y abandono en la infancia. Barcelona: Martínez Roca, 1993

MARTÍNEZ-SEGURA, María José. Deprivación socioambiental: Malos tratos en la infancia. Material de estudio, inédito fotocopiable para el uso de los alumnos. Cátedra de biopatología de la edad escolar. Universidad de Murcia, 2003, p.42.

MEJÍA, Sonia. Investigación sobre el maltrato infantil en Colombia 1985-1996. Estado del arte. Bogotá: ICBF- Fundación FES, 1996.

MIELES, María Dilia; HENRÍQUEZ, lliana; SÁNCHEZ, Ligia. Identidad personal y profesional de los docentes de preescolar en el Distrito de Santa Marta. Educación y Educadores, Bogotá: Universidad de la Sabana, v. 12, n. 1, 2009.

MEN. Ministerio Educación Nacional, Colombia. Política Educativa para la Primera Infancia. Atención del niño menor de cero a cuatro años. Bogotá, 2007.

OCHOA, Niévalo José. El maltrato infantil una respuesta de los padres al fracaso en un ideal de respuesta esperada por parte de los hijos. Bogotá: Universidad Javeriana, 2004.

OGBURN, William; NIMKOFF, Meyer Sociología. Grupo y personalidad. Bogotá: Aguilar, 1980.

ONU. Organización de las Naciones Unidas. Convención sobre los derechos del niño. 1989. Documento en línea. Disponible en: $<$ http://www.un.org/es/documents/>. Consulta: 10/02/2007.

. Fondo de Naciones Unidas para la Infancia. Las voces de niñas, niños y adolescentes. Consulta Latinoamericana sobre la violencia contra los niños. 2006. Documento en línea. Disponible en: < http://savethechildrendominicana.org/ pdf/consulta_latinoamericana_nna_estudio.pdf>. Consulta: 20/06/2009.

PARDO, Laura Marcela. Diagnóstico Preliminar del Maltrato Infantil en el Departamento de Sucre. Ponencia Primer encuentro interuniversitario Derechos de la niñez y juventud. Bogotá: Editora Guadalupe, 2003.

SANTANA-TAVIRA Rosalinda; SÁNCHEZ-AHEDO Roberto; HERRERA-BASTO, Emilio. El maltrato infantil: un problema mundial. Revista Salud Pública, México, v. 40, n. 1, p.58-65, 1998.

SPINETTA, Jean; RIGLER, David. The child abusing parent: A pychologycal review. Psychological Bulletin, Rockville Pike, USA, v. 77, p. 296-304, 1972.

SORIANO, Andrés. ¿Por qué maltratan los padres a sus hijos? Escuela y programas educativos en la educación primaria. 2008. Documento en línea. Disponible en: <dialnet.unirioja.es/servlet/fichero_articulo?codigo=2717076\&ord en $=0>$. Consulta: 28/06/2009.

SORIANO Francisco. Promoción del buen trato y prevención del maltrato en la infancia en el ámbito de la atención primaria de la salud. 2005. Documento en línea. Disponible en: <http://www.aepap.org/previnfad/pdfs/previnfad_maltrato. pdf. $>$. Consulta: 28/06/2011.

VIZCARRA, María Beatriz et al. Maltrato infantil en la ciudad de Temuco. Estudio de prevalencia y factores asociados. Revista médica de Chile, Santiago, ISSN 0034-9887, v. 129, n. 12, 2001. 
ZUNZUNEGUI, María Victoria.; MORALES, Manuel; MARTíNEZ, V. "Maltrato infantil: Factores socioeconómicos y estado de salud". In: Anales españoles de pediatría, Escuela Andaluza de Salud Pública, Vol. 47, nº 1, p. 33-41, 1997.

Recebido em: 22.12.2010

Aprovado em: 25.08 .2011

María Dilia Mieles Barrera es Directora DEL Grupo de Investigación en Educación Infantil. Magíster en Educación y estudiante de doctorado en Ciencias Sociales Niñez y Juventud. Docente Asociada Universidad del Magdalena (Santa Marta, Colombia) Email: mariadilia61@gmail.com

María Victoria Gaitán Espitia es miembro del Grupo de Investigación en Educación Infantil, Universidad del Magdalena. Licenciada en Psicopedagogía, Magíster en Orientación y Asesoría Educativa. Docente investigadora.

Renán Cepeda Gaitán es miembro del Grupo de Investigación en Educación Infantil. Comunicador y Periodista de la Universidad Sergio Arboleda. Investigador y Documentalista, estudiante de maestría en Comunicación Universidad Austral de Chile.Email: renhanc@hotmail.com 